\title{
ON THE LENGTH SPECTRUM METRIC IN INFINITE DIMENSIONAL TEICHMÜLLER SPACES
}

\author{
Erina Kinjo
}

Tokyo Institute of Technology, Department of Mathematical and Computing Sciences

Oh-okayama 2-12-1, Meguro-ku, Tokyo 152-8552, Japan; kinjo.e.aa@m.titech.ac.jp

\begin{abstract}
We consider the length spectrum metric $d_{L}$ in infinite dimensional Teichmüller space $T\left(R_{0}\right)$. It is known that $d_{L}$ defines the same topology as that of the Teichmüller metric $d_{T}$ on $T\left(R_{0}\right)$ if $R_{0}$ is a topologically finite Riemann surface. In 2003, Shiga proved that $d_{L}$ and $d_{T}$ define the same topology on $T\left(R_{0}\right)$ if $R_{0}$ is a topologically infinite Riemann surface which can be decomposed into pairs of pants such that the lengths of all their boundary components except punctures are uniformly bounded by some positive constants from above and below. In this paper, we extend Shiga's result to Teichmüller spaces of Riemann surfaces satisfying a certain geometric condition.
\end{abstract}

\section{Introduction}

Let $R_{0}$ be a Riemann surface of infinite topological type. We consider a pair $(R, f)$ of a Riemann surface $R$ and a quasiconformal mapping $f: R_{0} \rightarrow R$. Two such pairs $\left(R_{1}, f_{1}\right)$ and $\left(R_{2}, f_{2}\right)$ are called equivalent if $f_{2} \circ f_{1}^{-1}: R_{1} \rightarrow R_{2}$ is homotopic to some conformal mapping, where the homotopy map does not necessarily keep points of ideal boundary $\partial R_{0}$ fixed. We denote the equivalence class of $(R, f)$ by $[R, f]$. The set of all equivalence classes is called the Teichmüller space of $R_{0}$; we denote it by $T\left(R_{0}\right)$.

The Teichmüller space $T\left(R_{0}\right)$ has a complete metric $d_{T}$ called the Teichmüller metric which is defined by

$$
d_{T}\left(\left[R_{1}, f_{1}\right],\left[R_{2}, f_{2}\right]\right)=\inf _{f} \log K(f),
$$

where the infimum is taken over all quasiconformal mappings from $R_{1}$ to $R_{2}$ that is homotopic to $f_{2} \circ f_{1}^{-1}$ and $K(f)$ is the maximal dilatation of $f$.

We introduce another metric on $T\left(R_{0}\right)$. Let $\mathcal{C}\left(R_{0}\right)$ be the set of non-trivial and non-peripheral closed curves in $R_{0}$. We define the length spectrum metric $d_{L}$ by

$$
d_{L}\left(\left[R_{1}, f_{1}\right],\left[R_{2}, f_{2}\right]\right)=\sup _{\alpha \in \mathcal{C}\left(R_{0}\right)}\left|\log \frac{\ell_{R_{1}}\left(f_{1}(\alpha)\right)}{\ell_{R_{2}}\left(f_{2}(\alpha)\right)}\right|,
$$

where $\ell_{R_{i}}\left(f_{i}(\alpha)\right)$ is the hyperbolic length of the closed geodesic on $R_{i}$ which is freely homotopic to $f_{i}(\alpha)$.

Proposition 1.1. [15, Proposition 3.5] Let $\mathcal{S}\left(R_{0}\right)$ be the set of simple closed curves in $R_{0}$. Then

$$
d_{L}\left(\left[R_{1}, f_{1}\right],\left[R_{2}, f_{2}\right]\right)=\sup _{\alpha \in \mathcal{S}\left(R_{0}\right)}\left|\log \frac{\ell_{R_{1}}\left(f_{1}(\alpha)\right)}{\ell_{R_{2}}\left(f_{2}(\alpha)\right)}\right| .
$$

doi:10.5186/aasfm.2014.3925

2010 Mathematics Subject Classification: Primary 30F60; Secondary 32G15.

Key words: Length spectrum, Teichmüller metric, Riemann surface of infinite type. 
In 1972, Sorvali [14] defined $d_{L}$, and showed the following.

Lemma 1.2. [14] For any $\left[R_{1}, f_{1}\right],\left[R_{2}, f_{2}\right] \in T\left(R_{0}\right)$,

$$
d_{L}\left(\left[R_{1}, f_{1}\right],\left[R_{2}, f_{2}\right]\right) \leq d_{T}\left(\left[R_{1}, f_{1}\right],\left[R_{2}, f_{2}\right]\right)
$$

holds.

Sorvali conjectured that $d_{L}$ defines the same topolpgy as that of $d_{T}$ on $T\left(R_{0}\right)$ if $R_{0}$ is a topologically finite Riemann surface. In 1986, Li [9] proved that the statement holds in the case where $R_{0}$ is a compact Riemann surface with genus $\geq 2$. In 1999, Liu [10] proved that Sorvali's conjecture is true and he asked whether or not the statement holds for any Riemann surface of infinite type. To this question, Shiga [13] gave a negative answer, that is, he showed that there exists a Riemann surface $R_{0}$ of infinite type such that $d_{L}$ and $d_{T}$ do not define the same topology on $T\left(R_{0}\right)$. Also, he gave a sufficient condition for these metrics to define the same topology on $T\left(R_{0}\right)$ as follows.

Theorem 1.3. [13] Let $R_{0}$ be a Riemann surface. Assume that there exists a pants decomposition $R_{0}=\bigcup_{k=1}^{\infty} P_{k}$ satisfying the following conditions.

(1) Each connected component of $\partial P_{k}(k=1,2,3 \ldots)$ is either a puncture or a simple closed geodesic of $R_{0}$.

(2) There exists a constant $M>0$ such that if $\alpha$ is a boundary curve of some $P_{k}$ then

$$
0<M^{-1}<l_{R_{0}}(\alpha)<M
$$

holds.

Then $d_{L}$ defines the same topology as that of $d_{T}$ on $T\left(R_{0}\right)$.

In our previous paper [8], we showed that the converse of Shiga's theorem is not true, that is, there exists a Riemann surface $R_{0}$ such that $R_{0}$ does not satisfy Shiga's condition, but the two metrics define the same topology on $T\left(R_{0}\right)$. In this paper, we generalize the example and extend Shiga's theorem as follows.

Theorem 1.4. Let $R_{0}$ be a Riemann surface. Assume that there exists a constant $M>0$ and a decomposition $R_{0}=S \cup\left(R_{0}-S\right)$ such that

(1) $S$ is an open subset of $R_{0}$ whose relative boundary consists of simple closed geodesics and each connected component of $S$ has a pants decomposition satisfying the same condition as that of Shiga's theorem for $M$, and

(2) $R_{0}-S$ is of genus 0 and $d_{R_{0}}(x, S)<M$ for any $x \in R_{0}-S$, where $d_{R_{0}}(\cdot, \cdot)$ is the hyperbolic distance in $R_{0}$.

Then $d_{L}$ defines the same topology as that of $d_{T}$ on $T\left(R_{0}\right)$.

In Section 2, we show that there exists a Riemann surface such that it satisfies the condition of Theorem 1.4 but it does not satisfy that of Theorem 1.3. In Section 3, we introduce lemmas to prove Theorem 1.4. In Section 4, we prove Theorem 1.4.

In Section 5, we consider Riemann surfaces with bounded geometry. Here we say that a Riemann surface $R_{0}$ has $(M$-) bounded geometry if it satisfies the following condition: There exists a constant $M>0$ such that any closed geodesic has the length greater than $1 / M$ and for any $x \in R_{0}$, there exists a closed curve based on $x$ with the length less than $M$.

As a corollary of Theorem 1.4, we obtain the following: 
Corollary 1.5. Suppose that $R_{0}$ is of finite genus and $R_{0}$ has bounded geometry. Then $d_{L}$ define the same topology as that of $d_{T}$ on $T\left(R_{0}\right)$.

Remark 1. We consider surfaces after cutting the flares if such cylindrical ends exist. We assume that the unique complete hyperbolic metric on $R_{0} \backslash \partial R_{0}$ that uniformizes the complex structure on the surface satisfies the following condition: Let $\left\{P_{k}\right\}$ be a pants decomposition of $R_{0}$. If we replace each boundary component of $P_{k}(k=1,2, \ldots)$ with the closed geodesic in its homotopy class, then $P_{k}$ becomes a sphere with three holes, where a hole is either a boundary component which is a closed geodesic or a cusp.

Acknowledgement. The author would like to thank Professor Hiroshige Shiga and Professor Katsuhiko Matsuzaki for their valuable comments and suggestions.

\section{Examples}

First, we give examples of Riemann surfaces satisfying the conditions in Theorem 1.4 and Corollary 1.5.

Example 1. Any Riemann surface satisfying Shiga's condition satisfies the condition in Theorem 1.4. Hence, in particular, any Riemann surface of finite topological type satisfies it.

Example 2. The Riemann surface $R_{0}$ constructed in our previous paper [8] satisfies conditions in both Theorem 1.4 and Cororally 1.5. For convenience of the reader, we show the construction.

Let $\Gamma$ be a hyperbolic triangle group of signature $(2,4,8)$ acting on the unit disk $\mathrm{D}$ and let $P$ be a fundamental domain for $\Gamma$ with angles $(\pi, \pi / 4, \pi / 4, \pi / 4)$. (See the left in Figure 1.) Let $O, a, b, c$ denote the vertices of $P$, where the angle at $O$ is $\pi$. Now, take a sufficiently small number $\varepsilon>0$. Let $b^{\prime}$ the point on the segment $[O b]$ whose hyperbolic distance from $b$ is $\varepsilon$. Similarly, we take $a^{\prime}$ and $c^{\prime}$ in $P$. (See the middle in Figure 1.)

We define a Riemann surface $R_{0}$ by removing the $\Gamma$-orbits of $a^{\prime}, b^{\prime}, c^{\prime}$ from the unit disk D. (See the right in Figure 1.)

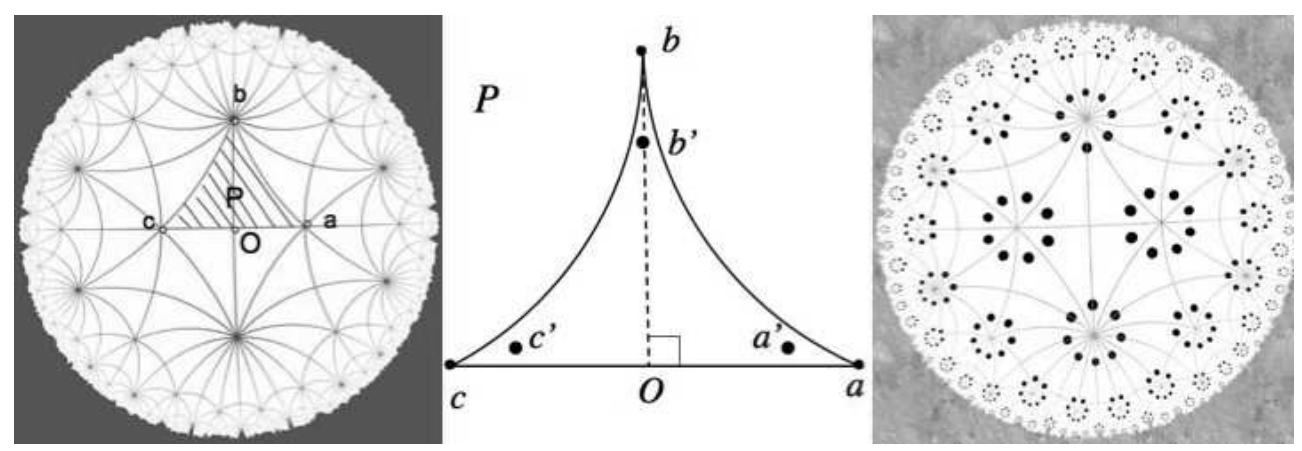

Figure 1. Left: Tessellation by the $(2,4,8)$ group. Middle: Points $a^{\prime}, b^{\prime}, c^{\prime}$ in $P$. Right: A Riemann surface $R_{0}=\mathbf{D}-\left\{\gamma\left(a^{\prime}\right), \gamma\left(b^{\prime}\right), \gamma\left(c^{\prime}\right) \mid \gamma \in \Gamma\right\}$.

It is not hard to see that the surface $R_{0}$ does not satisfy Shiga's condition (cf. Section 2 in [8]). However, it satisfies the above conditions. Indeed, we decompose $R_{0}$ into eight times punctured disks and a multiply-connected domain. (See Figure 2.) 


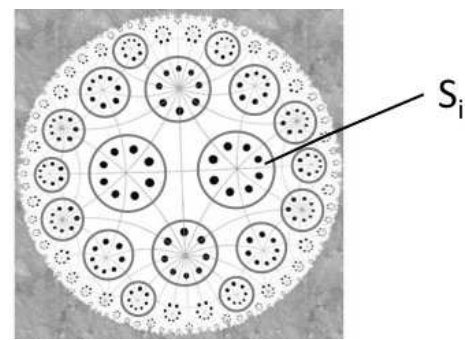

Figure 2. $R_{0}=\{$ punctured disks $\} \cup\{$ a multiply-connected domain $\}$.

Let $S_{i}$ be a punctured disk and put $S=\cup_{i=1}^{\infty} S_{i}$. Then we obtain a decomposition in Theorem 1.4: $R_{0}=S \cup\left(R_{0}-S\right)$. On the other hand, $R_{0}$ satisfies the condition in Corollary 1.5 obviously.

Also, we can construct a Riemann surfaces satisfying Theorem 1.4 and Corollary 1.5 by replacing a hyperbolic triangle group $\Gamma$ with an arbitrary Fuchsian group with a compact fundamental region.

Example 3. In Example 2, $R_{0}$ is a Riemann surface of genus 0 with $\infty$ punctures and 1 flare. By tinkering with $R_{0}$, we can construct Riemann surfaces of genus $\geq 1$ with two or more flares which satisfies the conditions of Theorem 1.4. For example, in Example 2, we replace a punctured disk $S_{i}$ with a pair of pants. (See the left in Figure 3.) We regard it as a block and make a copy of it and glue them. (See the right in Figure 3.) Then we obtain a Riemann surface $X_{0}$ of genus 1 with $\infty$ punctures and two flares. Obviously $X_{0}$ satisfies Theorem 1.4 and Corollary 1.5. Hence, in the similar way, we can construct Riemann surfaces of genus $\infty$ with $\infty$ flares which satisfies Theorem 1.4.
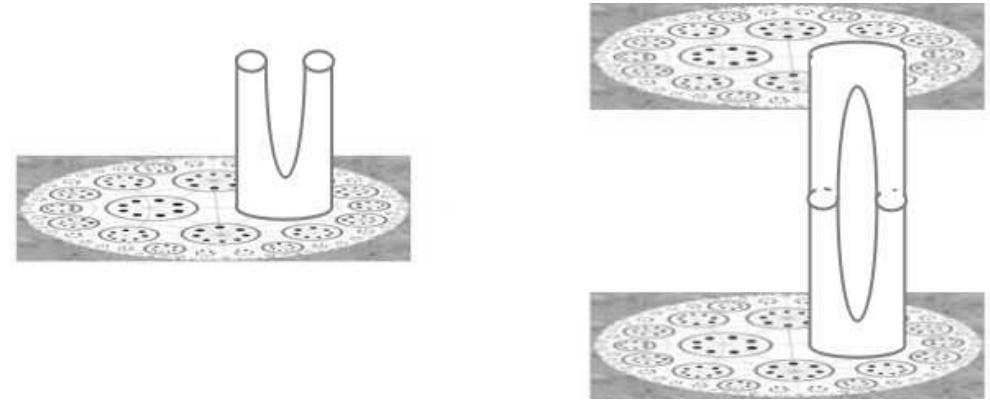

Figure 3. Left: A block obtained by replacing $S_{i}$ in Example 2 with a pair of pants. Right: A Riemann surface $X_{0}$ of genus 1 with $\infty$ punctures and two flares.

\section{Lemmas}

In this section, we present some lemmas to prove Theorem 1.4 and Corollary 1.5.

Lemma 3.1. [4, Lemma 3.1] Let $T_{1}, T_{2} \subset \mathbf{D}$ be two hyperbolic triangles with sides $\left(a_{1}, b_{1}, c_{1}\right)$ and $\left(a_{2}, b_{2}, c_{2}\right)$ respectively. Suppose all their angles are bounded below by $\theta>0$ and

$$
\varepsilon:=\max \left(\left|\log \frac{a_{1}}{a_{2}}\right|,\left|\log \frac{b_{1}}{b_{2}}\right|,\left|\log \frac{c_{1}}{c_{2}}\right|\right) \leq A .
$$

Then there is a constant $C=C(\theta, A)$ and a $(1+C \varepsilon)$-quasiconformal mapping $\varphi: T_{1} \rightarrow T_{2}$ such that $\varphi$ maps each vertex to the corresponding vertex and $\varphi$ is affine on the edge of $T_{1}$. 
Lemma 3.2. [4, Corollary 3.3] Let $H, H^{\prime} \subset \mathbf{D}$ be two hyperbolic hexagons with sides $\left(a_{1}, \ldots, a_{6}\right)$ and $\left(b_{1}, \ldots, b_{6}\right)$ respectively. Suppose $a_{1}, \ldots, a_{6}$ and $b_{1}, \ldots, b_{6}$ are $\leq B$ and are comparable with a constant $B$. Also assume that three alternating angles of $H$ and the corresponding angles of $H^{\prime}$ are $\pi / 2$ and the remaining angles are bounded below by $\theta>0$ and above by $\pi-\theta$. If $\varepsilon=\max _{i}\left|\log a_{i} / b_{i}\right| \leq 2$, then there is a constant $C=C(\theta, B)$ and a $(1+C \varepsilon)$-quasiconformal mapping $\varphi: H \rightarrow H^{\prime}$ such that $\varphi$ maps each vertex to the corresponding vertex and $\varphi$ is affine on the edge of $H$.

Lemma 3.3. [4, Lemma 6.2] Let $P_{1}$ and $P_{2}$ be pants with boundary lengths $\left(a_{1}, b_{1}, c_{1}\right)$ and $\left(a_{2}, b_{1}, c_{1}\right)$ respectively. Suppose $a_{1}, a_{2}, b_{1}, c_{1} \leq L$ (punctures count as length zero). Assume that $\varepsilon:=\left|\log a_{1} / a_{2}\right| \leq 2$, where we define $\left|\log a_{1} / a_{2}\right|=0$ if $a_{1}=a_{2}=0$ and $\left|\log a_{1} / a_{2}\right|=+\infty$ if one is zero and the other is not. Then there is a constant $C=C(L)$ and a $(1+C \varepsilon)$-quasiconformal mapping $\varphi: P_{1} \rightarrow P_{2}$ such that $\varphi$ is affine on each of the boundary components.

Also we note the following lemma.

Lemma 3.4. Let $R_{0}$ be a Riemann surface. Suppose $\alpha_{1}$ and $\alpha_{2}$ are disjoint simple closed geodesics. Let $\beta_{12}$ be a simple arc connecting $\alpha_{1}$ and $\alpha_{2}$. Then there exists a geodesic $\beta_{12}^{\star}$ connecting $\alpha_{1}$ and $\alpha_{2}$ such that

(1) $\beta_{12}$ and $\beta_{12}^{\star}$ are homotopic, where the homotopy map may not keep end points of $\beta_{12}$ and $\beta_{12}^{*}$ fixed;

(2) $\beta_{12}^{\star}$ is orthogonal to $\alpha_{1}$ and $\alpha_{2}$;

(3) the length of $\beta_{12}^{\star}$ is determined by lengths of three simple closed geodesics which are homotopic to $\alpha_{1}, \alpha_{2}$ and $\alpha_{12}:=\alpha_{1} \cdot \beta_{12} \cdot \alpha_{2} \cdot \beta_{12}^{-1}$.

Proof. There exists a closed geodesic in $R_{0}$ homotopic to $\alpha_{12}$. We denote it by $\left[\alpha_{12}\right]$. Consider a pair of pants $P_{12}$ bounded by $\alpha_{1}, \alpha_{2}$ and $\left[\alpha_{12}\right]$. There are three lines which divide $P_{12}$ into two isometric right-hexagons. Let $\beta_{12}^{\star}$ be a line connecting $\alpha_{1}$ and $\alpha_{2}$ in those. We denote the length of $\beta_{12}^{\star}$ by $\ell_{R_{0}}\left(\beta_{12}^{\star}\right)$. Then, by Theorem 7.19.2 of [3],

$$
\cosh \ell_{R_{0}}\left(\beta_{12}^{\star}\right)=\frac{\cosh \left(\frac{1}{2} \ell_{R_{0}}\left(\alpha_{12}\right)\right)+\cosh \left(\frac{1}{2} \ell_{R_{0}}\left(\alpha_{1}\right)\right) \cosh \left(\frac{1}{2} \ell_{R_{0}}\left(\alpha_{2}\right)\right)}{\sinh \left(\frac{1}{2} \ell_{R_{0}}\left(\alpha_{1}\right)\right) \sinh \left(\frac{1}{2} \ell_{R_{0}}\left(\alpha_{2}\right)\right)}
$$

holds.

In the following lemma, for $R_{0}-S$ in Theorem 1.4 we may consider a decomposition by right-hexagons with a bounded condition.

Lemma 3.5. Let $R_{0}$ be a Riemann surface satisfying the condition of Theorem 1.4. Then $R_{0}-S$ can be decomposed hyperbolic right-hexagons $\left\{H_{j}\right\}_{j=1}^{\infty}$ with sides of lengths less than $2 M$.

Proof. By assumption, we can decompose $S$ into domains $\left\{S_{i}\right\}_{i=0}^{\infty}$ such that $\partial S_{i} \cap\left(R_{0}-S\right)(i=0,1,2, \ldots)$ is a closed geodesic with the length less than $M$. For $S_{i}$, we consider the following domain:

$$
D_{i}:=\left\{x \in R_{0}-S \mid d_{R_{0}}\left(x, S_{i}\right) \leq d_{R_{0}}\left(x, S_{j}\right)(\forall j \neq i)\right\} .
$$

$D_{i}$ is contained in $M$-neighborhood of $S_{i}$, and $D_{i} \cup S_{i}$ is convex. The boundary of $D_{i}$ consists of two kinds of connected components; the boundary of $S_{i}$ and the boundary 
of geodesic polygon with finitely many sides. We denote the polygon with a hole by $W_{i}\left(\right.$ i.e. $\left.W_{i}:=\partial D_{i}\right)$.

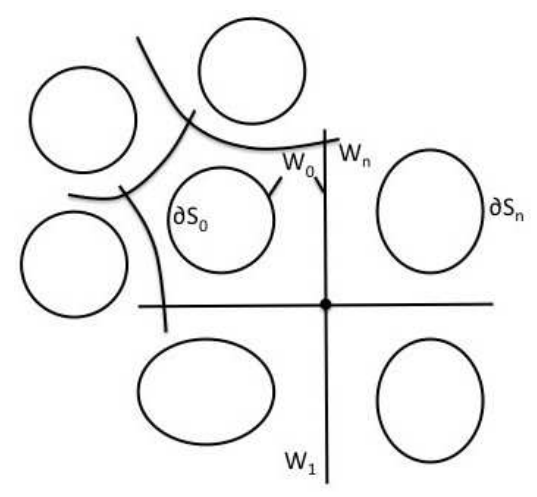

Figure $4 . W_{i}:=\partial D_{i}$.

$W_{i}$ is bounded on each side by another $W_{j}$. We show that two vertices of $W_{i}$ coincides with those of $W_{j}$ if $W_{i}$ is bounded by $W_{j}$. Assume that there exists a side $w_{i}$ with vertices $v_{i}, v_{i}^{\prime}$ of $W_{i}$ such that, for a side $w_{j}$ with vertices $v_{j}, v_{j}^{\prime}$ of $W_{j}, w_{j} \subset w_{i}$ but $0<d_{R_{0}}\left(v_{i}, v_{j}\right)<d_{R_{0}}\left(v_{i}, v_{j}^{\prime}\right)$. (See Figure 5.) Then there exists a polygon with a hole $W_{k}$ which has a side $w_{k} \cap w_{i}-w_{j} \neq \emptyset$. For a domain $S_{k}$ in $\left\{S_{i}\right\}_{i=0}^{\infty}$ with $\partial S_{k} \cap W_{k} \neq \emptyset$, we take a line $b_{i, k}:=\left\{x \in R_{0} \mid d_{R_{0}}\left(x, S_{i}\right)=d_{R_{0}}\left(x, S_{k}\right)\right\} . \quad b_{i, k}$ is a perpendicular bisector of the shortest geodesic segment $\left[l_{i, k}\right]$ connecting $\partial S_{i}$ and $\partial S_{k}$. (Note that $\left[l_{i, k}\right]$ is orthogonal to $\partial S_{i}$ and $\partial S_{k}$.) By the definition of domains $\left\{D_{i}\right\}, w_{k} \subset b_{i, k}$. Similarly we take another perpendicular bisector $b_{i, j}$ of the shortest geodesic segment $\left[l_{i, j}\right]$ connecting $\partial S_{i}$ and $\partial S_{j}$. Then $b_{i, k}=b_{i, j}$ since they are geodesics and $w_{k} \subset$ $w_{i} \subset b_{i, j}$. Take four points $p_{i, k}:=\partial S_{i} \cap\left[l_{i, k}\right], m_{i, k}:=\left[l_{i, k}\right] \cap b_{i, k}, p_{i, j}:=\partial S_{i} \cap\left[l_{i, j}\right]$, $m_{i, j}:=\left[l_{i, j}\right] \cap b_{i, j}$ and consider a quadrilateral with vertices $\left\{p_{i, k}, m_{i, k}, m_{i, j}, p_{i, j}\right\}$. Then we obtain a right-angled quadrilateral. However there does not exist such a hyperbolic quadrilateral. Hence $d_{R_{0}}\left(v_{i}, v_{j}\right)=0$, i.e. $v_{i}=v_{j}$. Similarly $v_{i}^{\prime}=v_{j}^{\prime}$.

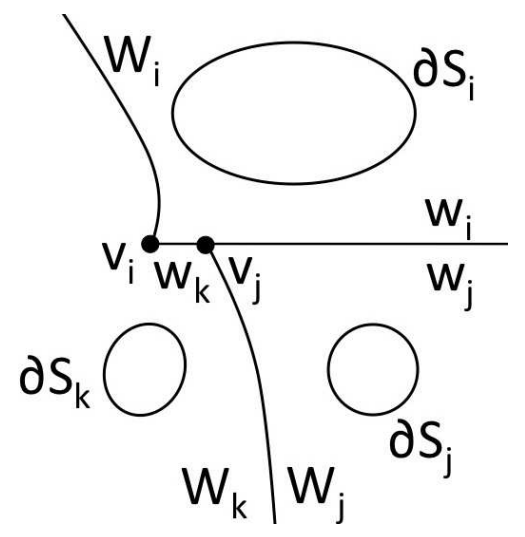

Figure 5. $v_{i} \neq v_{j}$.

Now, take an arbitrary vertex $v_{0}$ of an arbitrary polygon with a hole $W_{0}$ and put $W_{j}(j=0,1,2, \ldots, n$; in counterclockwise direction) the polygon with a hole which contains $v_{0}$. Connect $\partial S_{j}$ and $\partial S_{j+1}$ by the shortest geodesic segment $\left[l_{j, j+1}\right]$ for each $j=0,1,2, \ldots, n$ (, where $S_{j}$ is a domain in $\left\{S_{i}\right\}_{i=0}^{\infty}$ with $\partial S_{j} \cap W_{j} \neq \emptyset$ and $\left.S_{n+1}:=S_{0}\right)$. So we obtain a $2 n$-sided polygon $P$ which consists of $\left[l_{j, j+1}\right]$ and subarcs of $\partial S_{j}(j=0,1,2, \ldots, n)$. 
Also we join $\partial S_{0}$ and $\partial S_{j}(j=2,3, \ldots, n-1)$ by the shortest geodesic in $P$ which is orthogonal to $\partial S_{0}$ and $\partial S_{j}$, respectively. (By Lemma 3.4, there exist such geodesics.) Then we obtain $n-1$ right-hexagons. (See Figure 6.) Each right-hexagon has three alternating sides $\left[l_{0, j}\right],\left[l_{j, j+1}\right],\left[l_{j+1,0}\right]$ with the lengths bounded by $2 M$ since $\left[l_{j, j^{\prime}}\right] \leq d_{R_{0}}\left(v_{0}, \partial S_{j}\right)+d_{R_{0}}\left(v_{0}, \partial S_{j^{\prime}}\right)<M+M=2 M$ for $j, j^{\prime}=0,1,2, \ldots n, j \neq j^{\prime}$. Hence all lengths of sides are bounded by $2 M$.

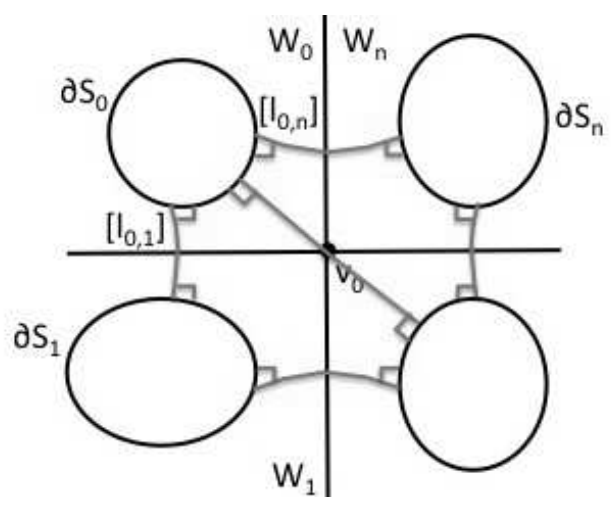

Figure 6. A right-hexagons decomposition around $v_{0}$.

Continue the above operation, then $R_{0}-S$ is divided into right-hexagons with sides of the lengths bounded by $2 M$.

\section{Proof of Theorem 1.4}

From Lemma 1.2, it is sufficient to show that for any sequence $\left\{p_{n}\right\}_{n=o}^{\infty} \subset T\left(R_{0}\right)$ with $d_{L}\left(p_{n}, p_{0}\right) \rightarrow 0(n \rightarrow \infty), d_{T}\left(p_{n}, p_{0}\right)$ converges to 0 as $n \rightarrow \infty$. We assume that $p_{0}=\left[R_{0}, i d\right]$. Put $p_{n}=\left[R_{n}, f_{n}\right]$.

In Section 3, we see that $R_{0}$ is decomposed by pairs of pants and hexagons such that lengths of their boundaries are bounded uniformly; $R_{0}=\bigcup_{i=1}^{\infty} S_{i} \cup \bigcup_{j=1}^{\infty} H_{j}$. We consider a decomposition of $R_{n}$ for sufficiently large $n$.

First, for each $j=1,2, \ldots$, we replace $f_{n}\left(H_{j}\right)$ by a right-hexagon in $R_{n}$ as follows. $H_{j} \subset R_{0}$ has edges $a_{1}, \ldots, a_{6}$ (in counterclockwise direction). We suppose that $a_{1}, a_{3}, a_{5}$ are subarcs of $\partial S_{1}, \partial S_{2}, \partial S_{3}$ respectively and $a_{2}$ connects $\partial S_{1}$ and $\partial S_{2}$. Put $\alpha_{12}:=\partial S_{1} \cdot a_{2} \cdot \partial S_{2} \cdot a_{2}^{-1} \in \mathcal{C}\left(R_{0}\right)$. For a closed curve $f_{n}\left(\alpha_{12}\right)$ in $R_{n}$, we take a closed geodesic $\left[f_{n}\left(\alpha_{12}\right)\right]$ in $R_{n}$. If we consider a pair of pants bounded by $\left[f_{n}\left(\partial S_{1}\right)\right]$, $\left[f_{n}\left(\partial S_{2}\right)\right]$ and $\left[f_{n}\left(\alpha_{12}\right)\right]$, then there exists a geodesic segment connecting $\left[f_{n}\left(\partial S_{1}\right)\right]$ and $\left[f_{n}\left(\partial S_{2}\right)\right]$ as in Lemma 3.4. We denote it by $a_{2}^{n}$. The length of $a_{2}^{n}$ is determined by the lengths of $\left[f_{n}\left(\partial S_{1}\right)\right],\left[f_{n}\left(\partial S_{2}\right)\right]$ and $\left[f_{n}\left(\alpha_{12}\right)\right]$. The lengths of them are almost the same as that of preimages of closed geodesics in $R_{0}$ respectively, so the lengths of $a_{2}$ and $a_{2}^{n}$ are almost the same. Similarly we take geodesic segments $a_{4}^{n}$ and $a_{6}^{n}$ in $R_{n}$ for $a_{4}$ and $a_{6}$ respectively. Let $H_{j}^{n} \subset R_{n}$ be a right-hexagon bounded by $a_{2}^{n}, a_{4}^{n}$, $a_{6}^{n}$ and subarcs of $\left[f_{n}\left(\partial S_{1}\right)\right],\left[f_{n}\left(\partial S_{2}\right)\right],\left[f_{n}\left(\partial S_{3}\right)\right]$. (See Figure 7.) Then $H_{j}^{n}$ is almost congruous with $H_{j}$.

Put $R_{n}^{\prime}:=\bigcup_{j=1}^{\infty} H_{j}^{n}$. By Lemma 3.2, we obtain a quasiconformal mapping $g_{n}$ from $R_{0}^{\prime}\left(=\bigcup_{j=1}^{\infty} H_{j}\right)$ to $R_{n}^{\prime}$. We claim that $f_{n}$ is homotopic to $g_{n}$ on $R_{0}^{\prime}$, where the homotopy map does not necessarily keep points of $\partial R_{0}$ fixed. 

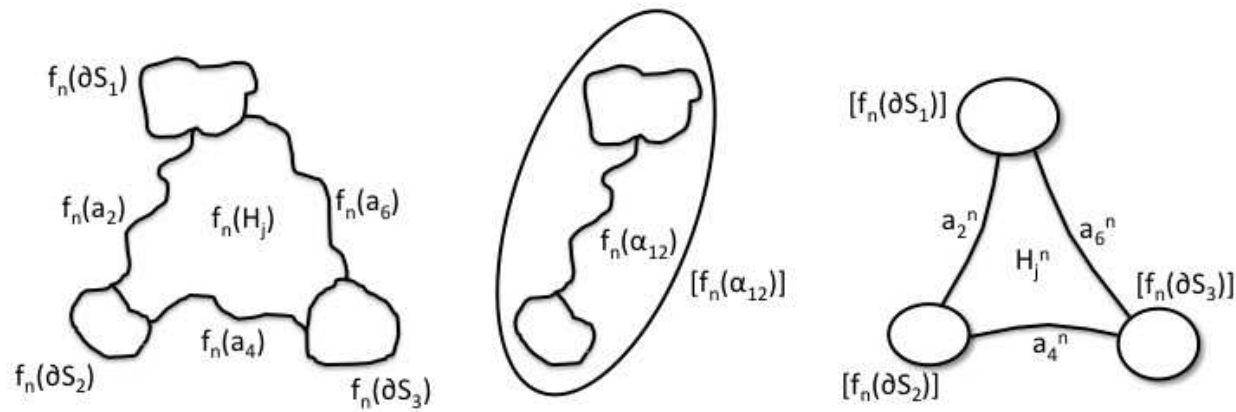

Figure 7. Replacement of $f_{n}\left(H_{j}\right)$ by a right-hexagon $H_{j}^{n}$ in $R_{n}$.

It is enough to see that for an arbitrary simple closed geodesic $\alpha \subset R_{0}^{\prime}, f_{n}(\alpha)$ and $g_{n}(\alpha)$ are homotopic (cf. [5, Lemma 4]). Let $\left\{H_{j(k)}\right\}_{k \in K} \subset R_{0}^{\prime}$ be the set of all the right-hexagons such that $H_{j(k)} \cap \alpha \neq \emptyset$. Since $f_{n}$ is homeomorphic, $f_{n}(\alpha) \subset$ $\bigcup_{k \in K} f_{n}\left(H_{j(k)}\right)$. Therefore we see that for each $k \in K$, a curve $g_{n}(\alpha) \cap H_{j(k)}^{n}$ is homotopic to a curve $\left[f_{n}(\alpha)\right] \cap H_{j(k)}^{n}$, where the homotopy map does not necessarily fix endpoints. (See Figure 8.) Hence $f_{n}(\alpha)$ is homotopic to $g_{n}(\alpha)$, so we verify the claim.

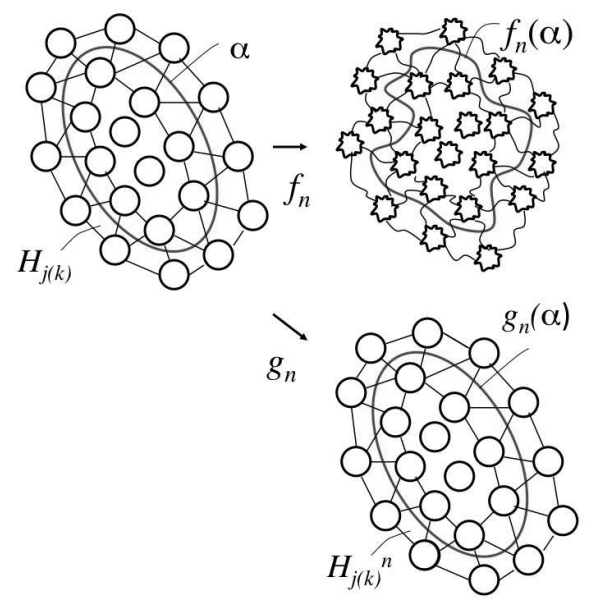

Figure 8. $f_{n}(\alpha)$ and $g_{n}(\alpha)$.

Next, we consider a quasiconformal mapping of $S_{i}$ for each $i=1,2, \ldots$ We decompose $S_{i}$ into pairs of pants satisfying Shiga's condition; $S_{i}=\bigcup_{k=1}^{\infty} P_{k}$.

Let $G_{P}$ be the set of closed geodesics which are boundaries of some $P_{k}$ in $S_{i}$. For each $\alpha \in G_{P}$, there exists a closed geodesic $\left[f_{n}(\alpha)\right]$ in $R_{n}$ homotopic to $f_{n}(\alpha)$. The set $\left\{\left[f_{n}(\alpha)\right]\right\}_{\alpha \in G_{P}}$ gives a pants decomposition of $f_{n}\left(S_{i}\right)$. (Indeed, $f_{n}\left(S_{i}\right)$ is Nielsenconvex; cf. [1, Theorem 4.5.])

Now, we put $\alpha_{1}, \alpha_{2}, \alpha_{3}$ three closed geodesics of $\partial P_{1}$ and assume that $\alpha_{1} \subset \overline{R_{0}^{\prime}}$.

By lemmas of Bishop, we obtain a quasiconformal mapping on $\overline{S_{i}-P_{1}}$. However, $g_{n}$ on $R_{0}^{\prime}$ is locally affine on $\alpha_{1}$, so we construct a quasiconformal mapping on $P_{1}$.

Let $x_{1}, \ldots, x_{m} \in \alpha_{1}$ be vertices of right-hexagons $\left\{H_{j}\right\}$, and let $y_{1}, \ldots, y_{6} \in \partial P_{1}$ be the vertices of two symmetric right-hexagons constructing $P_{1}$ (See Figure 9). Suppose that $y_{1}$ is on the segment $\left[x_{1} x_{2}\right]$, and $y_{6}$ is on the segment $\left[x_{r} x_{r+1}\right](1 \leq r \leq$ $m)$. Let $d_{1}$ be the length of $\left[x_{1} x_{2}\right]$ and let $d_{1}^{\prime}$ be the length of the $\left[x_{1} y_{1}\right]$. Then there is a number $t \in[0,1]$ such that $d_{1}=t d_{1}^{\prime}$. Similarly take $d_{r}, d_{r}^{\prime}$ for $\left[x_{r} x_{r+1}\right],\left[x_{r} y_{6}\right]$, then there is a number $s \in[0,1]$ such that $d_{r}=s d_{r}^{\prime}$. 


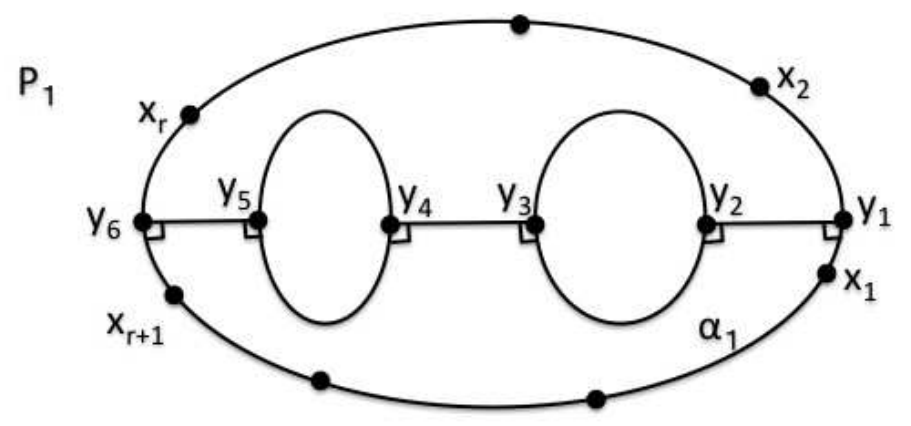

Figure 9. Points on $\partial P_{1}$.

On the other hand, let $x_{1}^{n}, \ldots, x_{m}^{n} \in\left[f_{n}\left(\alpha_{1}\right)\right]$ in $R_{n}$ be vertices of right-hexagons $H_{j}^{n}$. We take the points $g_{n}\left(y_{1}\right), \ldots, g_{n}\left(y_{6}\right)$ on $\partial P_{1}^{n}$, where $P_{1}^{n}$ is a pair of pants corresponding to $f_{n}\left(P_{1}\right)$

We consider a hyperbolic hexagon with vertices $g_{n}\left(y_{1}\right), \ldots, g_{n}\left(y_{6}\right)$. We claim that the angle formed by $\left[g_{n}\left(y_{2}\right) g_{n}\left(y_{3}\right)\right]$ and $\left[g_{n}\left(y_{3}\right) g_{n}\left(y_{4}\right)\right]$ is almost $\pi / 2$. Indeed, for $S_{i} \subset R_{0}$, let $\hat{S}_{i}$ be the Nielsen extension of $S_{i}$. We consider the Fenchel-Nielsen coordinates of the Teichmüller space $T\left(\hat{S}_{i}\right)$. Then the twist parameter along $\left[f_{n}\left(\alpha_{2}\right)\right]$ is almost the same as that along $\alpha_{2}$ (cf. [13, Lemma 4.1]). Hence we verify the claim. The remaining angles are almost $\pi / 2$, similarly.

Let $d_{i}^{n}$ be the hyperbolic length of the segment $\left[x_{i}^{n} x_{i+1}^{n}\right](1 \leq i \leq m)$, and let $d_{i}^{n \prime}$ be the hyperbolic length of the segment $\left[x_{i}^{n} g_{n}(*)\right]\left(i=1, r, *=y_{1}, y_{6}\right)$. Then, for $t \in[0,1]$ and $s \in[0,1]$ we took above, $d_{1}^{n \prime}=t d_{1}^{n}$ and $d_{r}^{n \prime}=s d_{r}^{n}$ hold, because $g_{n}$ of $R_{0}^{\prime}$ is locally affine on $\alpha_{1}$. Moreover, since the quasiconformal mapping $g_{n}$ of $\overline{S_{i}-P_{1}}$ is affine on $\alpha_{2}$ and $\alpha_{3}$, the lengths of sides $\left[y_{1} y_{2}\right], \ldots,\left[y_{6} y_{1}\right]$ and the lengths of sides $\left[g_{n}\left(y_{1}\right) g_{n}\left(y_{2}\right)\right], \ldots,\left[g_{n}\left(y_{6}\right) g_{n}\left(y_{1}\right)\right]$ are almost the same respectively. Hence the righthexagon with vertices $\left(y_{1}, \cdots, y_{6}\right)$ and the hexagon with vertices $\left(g_{n}\left(y_{1}\right), \cdots, g_{n}\left(y_{6}\right)\right)$ are almost congruous.
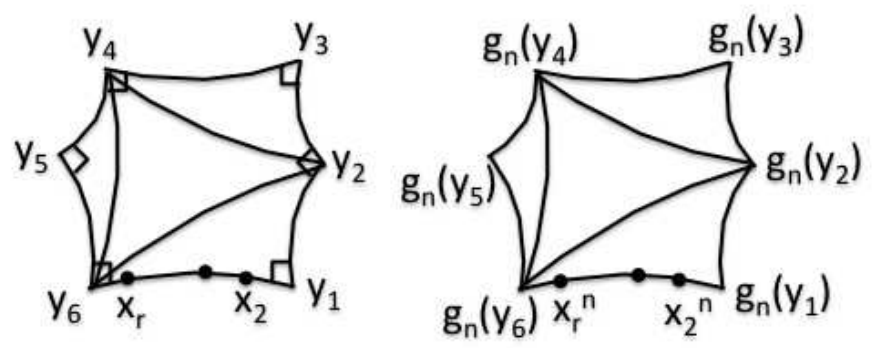

Figure 10. Triangulation.

From the First Cosine Rule for hyperbolic geometry (cf. [3]), the length of the new sides are determined by the sides and angles of the hexagon. Quasiconformal mappings of the triangles with vertices $\left(y_{2}, y_{3}, y_{4}\right),\left(y_{4}, y_{5}, y_{6}\right)$ and $\left(y_{2}, y_{4}, y_{6}\right)$ are obtained from Lemma 3.1.

We consider a quasiconformal mapping of the triangle $T$ with vertices $\left(y_{1}, y_{2}, y_{6}\right)$. In $T$, connect the points $x_{2}, \ldots, x_{r}$ by geodesics segments to $y_{2}$. Similarly, in the triangle $T_{n}$ with vertices $\left(g_{n}\left(y_{1}\right), g_{n}\left(y_{2}\right), g_{n}\left(y_{6}\right)\right)$, connect the points $x_{2}^{n}, \ldots, x_{r}^{n}$ by 
geodesics segments to $g_{n}\left(y_{2}\right)$. Then we obtain a quasiconformal mapping of the triangle $T$ from Lemma 3.1.

Hence we obtain a quasiconformal mapping $g_{n}$ of the whole of $R_{0}$ such that $g_{n}$ is homotopic to $f_{n}$ and $K\left(g_{n}\right) \rightarrow 1(n \rightarrow \infty)$. Thus $d_{T}\left(p_{n}, p_{0}\right) \rightarrow 0(n \rightarrow \infty)$.

In the case where $p_{0} \neq\left[R_{0}, i d\right]$, we can show that $d_{T}\left(p_{n}, p_{0}\right) \rightarrow 0(n \rightarrow \infty)$ similarly. Indeed, any Riemann surface which is quasiconformally equivalent to $R_{0}$ satisfies the condition of Theorem 1.4 for some constant.

\section{Corollary of Theorem $\mathbf{1 . 4}$}

In this section, we consider Riemann surfaces with bounded geometry.

Proposition 5.1. Let $R_{0}$ be a Riemann surface of finite genus with $M$-bounded geometry. Then $R_{0}$ satisfies the assumption of Theorem 1.4.

Proof. On $R_{0}$, we construct $S$ in the condition of Theorem 1.4 as a union of pairs of pants. Note that we may construct a pair of pants from two disjoint simple closed geodesics and an simple arc connecting the two geodesics.

At first, we take a constant $d=d(M)>0$ as the following:

For any $x \in R_{0}$, there exists a closed curve $c_{x}$ passing through $x$ with $1 / M<$ the length of $c_{x}<M$. We take a geodesic $\alpha_{x}$ in the homotopy class of $c_{x}$. We put

$$
d_{x}:=\max _{\alpha_{x}}\left\{M-\ell_{R_{0}}\left(\alpha_{x}\right)\right\}>0,
$$

where $\alpha_{x} \in\left\{\alpha_{x}\right.$ : a geodesic $\mid$ In the homotopy class of $\alpha_{x}$, there exists a closed curve $c_{x}$ passing through $x$ with $1 / M<$ the length of $c_{x}<M$. $\}$. Moreover we put

$$
d:=\sup _{x \in R_{0}} d_{x}
$$

Then $d$ satisfies the following property: For any $x \in R_{0}$ and any closed curve $c$ passing through $x$ with $1 / M<$ the length of $c<M$, the geodesic $\alpha$ in the homotopy class of $c$ satisfies $d_{R_{0}}(x, \alpha)<d$.

Indeed, if $d_{R_{0}}(x, \alpha) \geq d$ holds, then $d_{R_{0}}(x, \alpha) \geq d_{x} \geq M-\ell_{R_{0}}(\alpha)$ i.e. $d_{R_{0}}(x, \alpha)+$ $\ell_{R_{0}}(\alpha) \geq M$. Hence the length of $c\left(\geq d_{R_{0}}(x, \alpha)+\ell_{R_{0}}(\alpha)\right)$ is larger than $M$. This contradicts. Therefore $d_{R_{0}}(x, \alpha)<d$.

(Note that, in other words, $d$ is a constant such that if $x$ is a point in $R_{0}$ and $\alpha$ is a simple closed geodesic with $1 / M<\ell_{R_{0}}(\alpha)<M$ and $d_{R_{0}}(x, \alpha) \geq d$, then the length of any closed curve $c$ passing through $x$ which is homotopic to $\alpha$ is larger than $M$.)

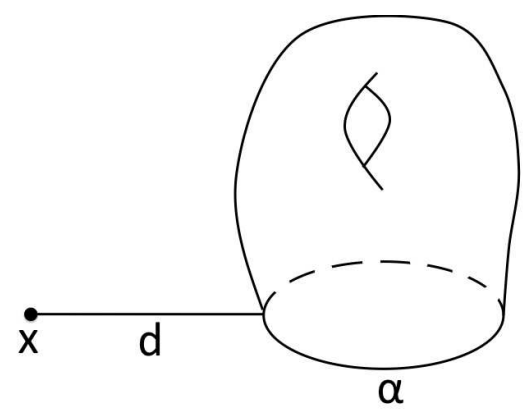

Figure 11. A closed curve $c$ such that the length $=d+\ell_{R_{0}}(\alpha)$.

Now let us start to construct pairs of pants. Let $x_{0}$ be an arbitrary point in $R_{0}$. Then there exists a closed curve $c_{0}$ passing through $x_{0}$ with the length less than $M$. 
We take a geodesic $\alpha_{0}$ in the homotopy class of $c_{0}$. Then $0<1 / M<\ell\left(\alpha_{0}\right)<M$ and $d_{R_{0}}\left(x_{0}, \alpha_{0}\right)<d$.

Put $D:=\max \{d, M\}$. Next, take $y_{0} \in R_{0}$ with $d_{R_{0}}\left(y_{0}, \alpha_{0}\right)=D+1$. Also, take a geodesic $\beta_{0}$ for $y_{0}$ in the above way. Then $\beta_{0} \neq \alpha_{0}$ and $d_{R_{0}}\left(y_{0}, \beta_{0}\right)<d$. Hence $d_{R_{0}}\left(\alpha_{0}, \beta_{0}\right)<D+1+d<2 D+1$. Thus there exists a simple arc $\hat{\gamma}_{0}$ connecting $\alpha_{0}$ and $\beta_{0}$ such that the length of $\hat{\gamma}_{0} \leq 2 D+1$.

If we construct a pair of pants $P_{0}$ by $\alpha_{0}, \beta_{0}$ and $\hat{\gamma}_{0}$, then the length of each boundary component is bounded by some constant $L=L(M)$ from above and below.

Next, we take a point $x_{1} \in R_{0}$ such that $d_{R_{0}}\left(P_{0}, x_{1}\right)=3 D+2$. Also, take a geodesic $\alpha_{1}$ for $x_{1}$ in the above way. (Note that $d_{R_{0}}\left(\alpha_{1}, x_{1}\right)<D$.) Then we can take a point $y_{1}$ in $R_{0}-P_{0}$ such that $d_{R_{0}}\left(y_{1}, \alpha_{1}\right)=D+1$. Indeed, since

$$
d_{R_{0}}\left(\alpha_{1}, P_{0}\right) \geq d_{R_{0}}\left(x_{1}, P_{0}\right)-d_{R_{0}}\left(\alpha_{1}, x_{1}\right) \geq 2 D+2,
$$

$\left\{y \in R_{0} \mid d_{R_{0}}\left(y, \alpha_{1}\right)=D+1\right\} \cap P_{0}=\emptyset$.

Now, we take a geodesic $\beta_{1}$ for $y_{1}$ similarly. $\beta_{1} \neq \alpha_{1}$ and $d_{R_{0}}\left(y_{1}, \beta_{1}\right)<d<D$ hold. Hence $d_{R_{0}}\left(\alpha_{1}, \beta_{1}\right)<2 D+1$. Thus $\beta_{1} \cap P_{0}=\emptyset$ since

$$
d_{R_{0}}\left(\beta_{1}, P_{0}\right) \geq d_{R_{0}}\left(P_{0}, \alpha_{1}\right)-d_{R_{0}}\left(\alpha_{1}, \beta_{1}\right) \geq 2 D+2-(2 D+1)=1 .
$$

Also, there exists a simple arc $\hat{\gamma_{1}}$ connecting $\alpha_{1}$ and $\beta_{1}$ with the length of $\hat{\gamma}_{1}<2 D+1$. Then we see that $\hat{\gamma_{1}} \cap P_{0}=\emptyset$ since $d_{R_{0}}\left(\alpha_{1}, P_{0}\right) \geq 2 D+2$.

We construct a pair of pants $P_{1}$ by $\alpha_{1}, \beta_{1}$ and $\hat{\gamma}_{1}$. Then $P_{0} \cap P_{1}=\emptyset$. Indeed, if we take a geodesic $\gamma_{1}$ which is homotopic to a closed curve $\alpha_{1} \cdot \hat{\gamma}_{1} \cdot \beta_{1} \cdot \hat{\gamma}^{-1}$ then $\gamma_{1} \cap P_{0}=\emptyset$ by property of geodesics.

Similarly, we take a point $x_{2} \in R_{0}$ such that $d_{R_{0}}\left(x_{2}, P_{0} \cup P_{1}\right)=3 D+2$. Let $\alpha_{2}$ be the geodesic in homotopy class of a simple closed curve $c_{2}$ passing through $x_{2}$ with $M^{-1}<$ the length of $c_{2}<M . d_{R_{0}}\left(\alpha_{2}, x_{2}\right)<d<D$. We can take a point $y_{2} \in R_{0}-\left(P_{0} \cup P_{1}\right)$ such that $d_{R_{0}}\left(\alpha_{2}, y_{2}\right)=D+1$ since $d_{R_{0}}\left(\alpha_{2}, P_{0} \cup P_{1}\right) \geq 2 D+2$. Let $\beta_{2}$ be the geodesic in homotopy class of a simple closed curve $c_{2}^{\prime}$ passing through $y_{2}$ with $M^{-1}<$ the length of $c_{2}^{\prime}<M . d_{R_{0}}\left(\beta_{2}, y_{2}\right)<d<D$. Hence $d_{R_{0}}\left(\alpha_{2}, \beta_{2}\right)<2 D+1$. Thus $\beta_{2} \cap\left(P_{0} \cup P_{1}\right)=\emptyset$. Also, there exists a simple arc $\hat{\gamma}_{2}$ connecting $\alpha_{2}$ and $\beta_{2}$ with $\ell\left(\hat{\gamma_{2}}\right)<2 D+1 . \hat{\gamma_{2}} \cap\left(P_{0} \cup P_{1}\right)=\emptyset$ since $d_{R_{0}}\left(\alpha_{2}, P_{0} \cup P_{1}\right) \geq 2 D+2$. If we construct a pair of pants $P_{2}$ by $\alpha_{2}, \beta_{2}$ and $\hat{\gamma_{2}}$ then $P_{2} \cap\left(P_{0} \cup P_{1}\right)=\emptyset$.

Inductively, we construct a sequence of pairs of pants $\left\{P_{i}\right\}$. Then it satisfies the following.

(i) $P_{i} \cap P_{j}=\emptyset$ if $i \neq j$.

(ii) For any $i$, the length of each connected component of $\partial P_{i}$ is bounded by $L$ from above and below.

Finally we consider the set

$$
X_{n}:=\left\{x \in R_{0} \mid d_{R_{0}}\left(x_{0}, x\right)<n\right\}
$$

for a sufficient large number $n>0$. Since the closure $\overline{X_{n}}$ of $X_{n}$ is relatively compact in $R_{0}$, there exists a finite sequence of pairs of pants $\left\{P_{i}\right\}_{i=0}^{k}$ such that $X_{n} \cap P_{i} \neq \emptyset$ $(i=0,1, \ldots, k)$ and it satisfies (i) and (ii); moreover

$$
d_{R_{0}}\left(x, \bigcup_{i=0}^{k} P_{i}\right)<3 D+2
$$

for any $x \in X_{n}-\bigcup_{i=0}^{k} P_{i}$. 
Construct $\left\{P_{i}\right\}_{i=0}^{\infty}$ as $n \rightarrow \infty$. Since $\bigcup_{n=1}^{\infty} X_{n}=R_{0}$, we obtain a subset $S=$ $\bigcup_{i=0}^{\infty} P_{i} \subset R_{0}$ in the condition of Theorem 1.4.

(We note that $R_{0}-S$ is of genus 0 . Indeed, the lengths of geodesics cutting genus of $R_{0}$ are bounded by $M$ since $R_{0}$ is of finite genus. Hence we can choose them as the curves of $\partial P_{i}$.)

Hence we obtain Corollary 1.5.

\section{References}

[1] Alessandrini, D., L. Liu, A. Papadopoulos, W. Su, and Z. Sun: On Fenchel-Nielsen coordinates on Teichmüller spaces of surfaces of infinite type. - Ann. Acad. Sci. Fenn. Math. 36, 2011, 621-659.

[2] Basmajian, A.: Hyperbolic structures for surfaces of infinite type. - Trans. Amer. Math. Soc. $336: 1,1993,421-444$.

[3] Beardon, A. F.: The geometry of discrete groups. - Springer-Verlag, New York, 1983.

[4] Bishop, C. J.: Quasiconformal mapping of Y-pieces. - Rev. Mat. Iberoamericana 18, 2002, $627-652$.

[5] Epstein, A.: Effectiveness of Teichmüller modular groups. - In: In the tradition of Ahlfors and Bers, Contemp. Math. 256, Amer. Math. Soc., 2000, 69-74.

[6] Fujikawa, E., H. Shiga, and M. Taniguchi: On the action of the mapping class group for Riemann surfaces of infinite type. - J. Math. Soc. Japan 56:4, 2004, 1069-1086.

[7] Fujikawa, E., and K. Matsuzaki: Stable quasiconformal mapping class groups and asymptotic Teichmüller spaces. - Amer. J. Math. 133, 2011, 637-675.

[8] KInJo, E.: On Teichmüller metric and the length spectrums of topologically infinite Riemann surfaces. - Kodai Math. J. 34, 2011, 179-190.

[9] LI, Z.: Teichmüller metric and length spectrums of Riemann surfaces. - Sci. Sinica (Ser. A) 29, 1986, 265-274.

[10] LiU, L.: On the length spectrums of non-compact Riemann surfaces. - Ann. Acad. Sci. Fenn. Math. 24, 1999, 11-22.

[11] Liu, L., and A. Papadopoulos: Some metrics on Teichmüller spaces of surfaces of infinite type. - arXiv:0808.0870v2.

[12] Liu, L., Z. Sun, and H. Wei: Topological equivalence of metrics in Teichmüller space. - Ann. Acad. Sci. Fenn. Math. 33, 2008, 159-170.

[13] Shiga, H.: On a distance by the length spectrum on Teichmüller space. - Ann. Acad. Sci. Fenn. Math. 28, 2003, 315-326.

[14] SoRvali, T.: The boundary mapping induced by an isomorphism of covering groups. - Ann. Acad. Sci. Fenn. Ser. A I Math. 526, 1972, 1-31.

[15] Thurston, W. P.: Minimal stretch maps between hyperbolic surfaces. - 1986 preprint converted to 1998 eprint, http://arxiv.org/pdf/math/9801039. 